\section{De cómo las normas sanitarias y de inocuidad de los alimentos atentan contra la producción campesina en el Ecuador}

Elizabeth Bravo*

ResUMen

En el Ecuador de los últimos años, Se ha impuesto una serie de normativas de carácter sanitario FITOSANITARIO, CUYA APLICACIÓN AFECTA GRANDEMENTE A LA PRODUCCIÓN CAMPESINA Y ARTESANAL. EN EST ARTíCULO SE HACE UNA REVISÍ́N SOBRE EL ORIGEN DE ESTAS NORMAS A NIVEL INTERNACIONAL, FUERTEMENT ARRAIGADAS A LAS NORMAS DE LIBRE COMERCIO, Y CUYO OBJETIVO ESTÁ ENCAMINADO A FORTALECER EL SISTEM AGROALIMENTARIO MUNDIAL, DESPLAZANDO LA PEQUEÑa PRODUCCIÓN. SE HACE UN BREVE ANÁLISIS DE LA SITUACIÓN DEL ECUADOR EN ESTA MATERIA, CON ESPECIAL ATENCION A LA PRODUCCION DE LACTEOS.

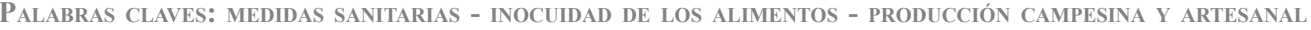
QUESERIAS.

Abstract

IN RECENT YEARS IN ECUADOR YEARS, A SET OF SANITARY AND PHYTOSANITARY REGULATIONS HAS BEEN IMPOSED WHOSE APPLICATION GREATLY AFFECTS ARTISANAL PRODUCTIVITY. IN THIS ARTICLE WE MAKE A REVIEW OF THE ORIGIY OF THESE NORMS AT THE INTERNATIONAL LEVEL, STRONGLY ROOTED TO FREE TRADE RULES, WHOSE GOAL AIMS TO REIFORCE THE GLOBAL AGRI-FOOD SYSTEM, DISPLACING SMALL AGRICULTURE PRODUCTION, A BRIEF ANALYSIS IS MADE OF ECUADOR's SITUATION IN THIS RESPECT, WITH SPECIAL ATTENTION GIVEN TO DAIRY PRODUCTS.

KEYWORDS: SANITARY MEASURES - FOOD SAFETY - PEISAYT AND ARTISAYAL PRODLCTION - DAIRIES.

* Universidad Politécnica Salesiana. Grupo de Investigación en Ecología Política. Correo electrónico: mariaelizabethbravovelasquez@gmail.com

\section{Introducción}

Z Ecuador, el $88 \%$ de las unidades productivas pertenecen a la agricultura campesina

y familiar. Este tipo de producción agrupa al 56\% del total de agricultores del país, y en ellas se produce la mitad de las hortalizas que se consume a nivel nacional, el $45 \%$ del maíz, un tercio de los cereales y el $30 \%$ del arroz. Dada la falta de acceso a créditos y otras ayudas, el uso de insumos externos (incluyendo pesticidas y fertilizantes) es mínima (Heifer Ecuador, 2014).

Esta forma de agricultura está adaptada a las realidades culturales y ambientales locales. Sus semillas evolucionan con los cambios que se dan en sus territorios, incluyendo a las alteraciones climáticas; y sobre todo, producen comida que está en armonía con las tradiciones de la población; por eso continúa siendo la principal fuente de alimentos para la población, no sólo en Ecuador, sino también en otros lugares del mundo (GRAIN, 2014)

Este es el caso de la producción del queso artesanal, una de las actividades agropecuarias en las que los pequeños productores están mejor preparados para competir con la gran industria láctea. La producción lechera a pequeña escala es un componente importante de la soberanía alimentaria local y nacional. A partir de ésta, se satisface las necesidades de la familia al tiempo que su excedente puede ser comercializado.

La pequeña ganadería es una actividad que genera empleo. Las lecherías generan mucha mano de obra, especialmente femenina. De acuerdo a datos de la FAO, por cada 100 litros de leche producida, se genera la siguiente cantidad de directos e indirectos: Bangladesh: 14,4, Ghana: 17,2, Kenia: 3,7. El informe señala además que la producción láctea es una fuente de ingreso para las familias (FAO/ILRI, 2004).

En muchos países quienes toman las decisiones en la cría de ganado lechero son las mujeres. Estas decisiones tienen que ver con la comercialización, con el uso que se da a los excedentes generados, generalmente utilizados para cubrir las necesidades básicas de la familia que no son obtenidos en la unidad productiva, etc.

A pesar de su importancia, las queserías rurales están en peligro, ya que desde hace algunos años el gobierno ha empezado a imponer una serie de requisitos de carácter técnico y administrativo, que para una familia que tiene entre 3 a 10 cabezas de ganado, y una producción de entre 3 a 10 libras de queso al día, son imposibles de cumplir

Esta es una tendencia mundial que obedece al proceso de surgimiento y consolidación del sistema agroalimentario industrial que se inició a mediados del siglo XX (Friedmann, 2009), y que al momento se estima que mueve miles de millones de dólares anuales. En su proceso de consolidación ha tenido que desplazar la producción campesina, ya sea ocupando físicamente sus territorios (necesarios para la producción a gran escala, requisito indispensable para su expansión), o subordinándola a ella.

La producción campesina y la agroindustrial están en disputa. Cada actor social construye su propio territorio, utilizando sus propias tecnologías para dominarlo, construirlo o reconstruirlo (reterritorialización). Son espacios de conservación o de desperdicio (Schneider, 2009: 71-72).

En ese proceso se han conformado paisajes rurales del agronegocio (homogéneos, de compasión uniforme y geométrica con poca población rural, porque está ocupado por monocultivos destinados al mercado internacional o a la transformación agroindustrial); paisajes campesinos (diversos, con comunidades vivas; donde se produce alimentos para la soberanía alimentaria, y donde construyen su cultura y su tejido social). Hay además algunos territorios campesinos que están controlados por el agronegocio, pues la ocupación territorial está determinada, en términos de tecnología, tipo de producción y canales de comercialización, por una lógica empresarial (Fernandes, 2008).

El control sobre un territorio nunca es absoluto. No hay una dominación completa en las disputas por el control de un territorio; siempre quedan lagunas que dan lugar a mecanismos de adaptación o resistencia por parte de las comunidades (Vallejo, 2005: 3), y es en esas lagunas 
donde tienen lugar una serie de interacciones -casi siempre de tipo inequitativas-, es donde se ponen de manifiesto relaciones de poder

Con el fin de desplazar o controlar la producción campesina, la industria agroalimenticia ha diseñado estrategias de carácter tecnológico (como el desarrollo de semillas híbridas y transgénicas atadas a un paquete tecnológico producido generalmente por las mismas empresas, la hiper-industrialización de los procesos agrícolas y de transformación de los alimentos). La otra estrategia ha sido incidir en el desarrollo de políticas públicas y leyes que les favorezcan. Para ello, desarrollan un sistema de fuerte cabildeo y presión a los estados nacionales y las instancias internacionales pertinentes.

En este artículo, se analiza las normas que se han desarrollado para favorecer a la industria láctea, tanto a nivel internacional como del Ecuador; cuáles son los grupos de poder que se benefician de ellas; y cuáles son las implicaciones de su aplicación en la producción campesina y artesanal de queso en el país.

\section{Las medidas sanitarias, fitosanitarias y de inocuidad de los alimentos en la} normativa internacional

A lo largo de los años, se han venido desarrollando a nivel internacional una serie de normas, guías y estándares relacionados con la inocuidad de los alimentos y la sanidad animal y vegetal. En principio, su objetivo es el de garantizar que el comercio internacional de productos alimenticios, agrícolas, ganaderos y pesqueros sean seguros para el consumo humano; aunque en alimenticios, agrícolas, ganaderos y pesqueros sean seguros para el consumo humano; aunque en de los grupos económicos relacionados con la industria agroalimentaria.

Las normativas sobre medidas sanitarias, fitosanitarias, inocuidad de los alimentos y de buenas prácticas de manufactura surgen como una necesidad del creciente comercio internacional de alimentos, que cada vez está más concentrado en pocas empresas transnacionales.

En su esfuerzo por desplazar la producción independiente de alimentos, los Estados, desarrollaron a lo largo del siglo XX, un corpus legal internacional muy sofisticado, con el fin de beneficiar a sus empresas. Su aplicación afecta no sólo a los pequeños productos, sino también a empresas de los países económicamente débiles y con poca dotación de poder en el escenario internacional. Por ejemplo, en Colombia tanto la industria láctea nacional como la pequeña producción, se han afectado mucho a raíz de la adopción de tratados comerciales que, entre otras exigencias, incluyen medidas sanitarias muy estrictas, a lo que se suma la apertura con cero aranceles a productos lácteos (Espinosa, 2013).

A continuación se hace una revisión de los principales acuerdos internacionales que trabajan en el tema.

\section{Acuerdo de Medidas Sanitarias en la Organización Mundial de Comercio}

El Acuerdo de Medidas Sanitarias y Fitosanitarias (AMSF)² de la Organización Mundial de Comercio (OMC), es el acuerdo mas importante a nivel internacional en materia sanitaria. Los mecanismos establecidos por la Organizacion Mundial de Comercio permiten tomar medidas de

Las relaciones de poder pueden darse entre bloques económicos (ej. Primer/Tercer Mundo), sectores de la sociedad (ej. burguesia//lase acceso de los recursos naturales y productivos es materia de la Ecología Política (Bryant y Bailey, 2005). En este internacional $y$ que han sido capaces de insidiar en las normas que lo rigen y aquellas que se subordinan a las mimas (entre las que se encuentra el Ecuador); b) las relaciones que se dan entre empresas -que cuentan con el apoyo de los estados y su propio poder económico, y el campesinado.

El Acuerdo no se aplica a las medidas de protección del medio ambiente o de los intereses de los consumidores, n de defensa de los animales, las que son tratadas bajo el acuerdo de Obstáculos Técnicos al Comercio y el GATT de 994 en su artículo XX. retaliación económica que en la práctica, son usadas por países con mayor dotación de poder, en contra de otros que son económicamente más débiles.

Este acuerdo establece que un país o un bloque de países pueden imponer a otros ciertas restricciones a su comercio internacional, con el fin de precautelar la inocuidad de los alimentos, la sanidad de los animales y de los vegetales, para proteger la salud y la vida de las personas. Y si surgen divergencias, los países pueden acudir al mecanismo de resolución de controversias de la OMC. En esos casos se recomienda que primero se trate de alcanzar soluciones por la vía bilateral. Si se encuentra que un país no cumplió con sus obligaciones, se recomienda que éste deponga sus medidas, caso contrario, puede tomar retaliaciones económicas si estas disputas no llegaran a resolverse satisfactoriamente.

Aunque teóricamente el objetivo del acuerdo es precautelar la salud humana, la prioridad es el comercio internacional, pues los principios en los que se sustenta la $\mathrm{OMC}$ establecen que ninguna $\mathrm{MSF}^{3}$ puede convertirse en un obstáculo encubierto al comercio internacional.

\section{El consenso científico y la evaluación de riesgo}

El criterio para dirimir controversias en temas sanitarios es el consenso científico, lo que teóricamente podría pensarse que es justo, para que los países no tomen injustificadamente decisiones que frenen el comercio internacional de un producto y se constituya en una traba comercial.

Pero, en este análisis hay que recordar la gran disparidad en cuanto a capacidades científicas y tecnológicas de los distintos estados miembros de la OMC. De esa manera, si Estados Unidos pone trabas a un producto ecuatoriano por motivos sanitarios usando argumentos científicos; el Ecuador debe refutarlos con base en la ciencia. Pero su argumentación podría no ser aceptada dado que la ciencia del "Tercer Mundo" es poco valorada. Al respecto Douglas (1996), sostiene que la noción de riesgo depende de las percepciones que tiene una sociedad de otra y de un grupo de la sociedad a otro.

Un ejemplo paradigmático es la disputa entre la Unión Europea y Estados Unidos por la prohibición europea a la importación de carne tratada con hormonas. Por su parte, Estados Unidos prohíbe la importación de queso hecho con leche no pasterizada. Y aunque Estados Unidos fue el primer país en percibir la amenaza de los contaminantes químicos, en avizorar su potencial cancerígeno y en desarrollar sistemas de evaluación de riesgos en torno a esta problemática, hoy la percepción del riesgo ha cambiado en ese país, pues se teme más a la contaminación biológica (que podría estar presente en la leche sin pasterizar), que a la producida por las hormonas sintéticas. Este es un cambio de percepción de carácter puramente ideológico, promovido por la industria de alimentos, que está muy imbricada con la industria de los agrotóxicos.

La evaluación de riesgo es la metodología aceptada para evaluar la legitimidad de las medidas sanitarias o fitosanitaria adoptada por los Estado, la misma que se base en la probabilidad de que un evento ocurra, usando criterios objetivos y subjetivos; las decisiones que se toman son de carácter político, especialmente cuando surgen incertidumbres. ¿Cuáles son las metodologías y los principios epistemológicos para establecer que un insecto puede convertirse en una peste, o que un microorganismo se transforme en un patógeno, o que un alimento transgénico puede desencadenar impactos negativos a la salud?

La evaluación de riesgo puede arrojar resultados muy diversos porque trabaja con factores aislados, sin establecer interconexiones entre ellos, aun cuando determinado producto puede interactuar con otros factores; por eso, la evaluación de riesgos tiene límites para resolver

3 MSF: Medida Sanitaria o Fitosanitaria 
incertidumbres, aún más en el caso de las nuevas tecnologías alimenticias (alimentos transgénicos o nanotecnológicos). Al respecto, el AMSF dice que cuando la información científica es insuficiente para aceptar la importación de estos productos, los países pueden adoptar medidas provisionales sobre la base de la información existente, y en un tiempo razonable, buscar información adicional para poder hacer una evaluación de riesgo más objetiva (AMSF, Artículo 5.7). Pero, ¿qué es un tiempo razonable?

La evaluación de riesgo analiza además los umbrales de aceptabilidad de riesgo; es decir los riesgos que la sociedad está dispuesta asumir y el grado de tolerancia frente al riesgo. Ahora bien, la "sociedad" está conformada por actores muy diversos, cada uno de ellos con diversos tipos de intereses. La percepción de riesgo en relación a un alimento no va a ser igual para un habitante de una zona periurbana que para el gerente de una empresa alimenticia Adicionalmente, la aceptabilidad del riesgo depende de las clases sociales; la distribución del riesgo que está relacionada con la desigualdad social, pues a mayor pobreza, la tolerancia a riesgo aumenta (Douglas, 1996). Pero es la opinión del sector con mayor dotación de poder la que prevalece (Pavone y Arias, 2012), en este caso, la industria láctea transnacional4. Las formas de percibir los riesgos se ordenan sobre códigos y privilegios. El púbico percibe de una manera diferente los riesgos, que la percepción de un experto, que es quien los codifica, y éste privilegia los códigos técnicos.

Por eso, cuando hablamos del riesgo que una sociedad está dispuesta a correr, debemos preguntarnos de qué sociedad hablamos. En una sociedad de clases, donde imperan varios tipos de desigualdades, la "sociedad" es en realidad la clase dominante (Bravo, 2014). Las decisiones que un Estado tome en relación a la importación o exportación de un producto que puede entrañar problemas de sanidad animal y vegetal, va a reflejar las presiones que ejercen los grupos que controlan el sector agroalimentario en ese país.

Lo mismo sucede con la producción local. Aun cuando el consumo de un alimento hecho artesanalmente y con tecnologías tradicionales, puede no ser riesgoso; una empresa, que hace el mismo tipo de alimento de manera industrial, puede argumentar (y demostra científicamente) que el producto artesanal es peligroso y así poder desplazarlo. Este es el caso del queso artesanal, que como se verá con más detenimiento posteriormente, está seriamente amenazado por la aplicación de normas sanitarias.

En este análisis hay que tomar en cuenta que un país con alta dependencia económica hacia otro, difícilmente va a estar en condiciones de objetar la entrada de productos agrícola a su país; por razones sanitarias, y si eso ocurre, las controversias se resuelven bilateralmente, puesto que un Estado fuerte puede amenazar a otro con retaliaciones. Son muy raros los casos que pasan a los paneles de arbitraje. Pero por ejemplo en controversias como la de hormonas en la carne, donde se enfrentó Estados Unidos con la Unión Europea, las controversias toman mucho más tiempo en resolverse. Una controversia que ha durado muchos años y aún está pendiente, es la interpuesta por Estados Unidos a la Unión Europea por la moratoria de facto hecha a los alimentos transgénicos.

Entre 1996 y 1998 el APHIS ${ }^{5}$ reportó 112 restricciones a sus importaciones, por motivos de salud animal y vegetal, las que fueron resueltas a través de negociaciones bilaterales. Por su parte, la Secretaría de Agricultura de Estados Unidos identificó que en 1996 se habían impuesto 302 barreras "cuestionables" a sus exportaciones estadounidenses, relacionadas con temas sanitarios. Estas provenían de 61 países y dos bloques económicos y tenían un valor de 4,9 billones de dólares (Thornsburry, 2002).

4 Las 10 principales industrias lácteas a nivel mundial son Nestlé (Suiza), Danone y Groupe Lactalis (Francia) Fonterra (Nueva Zelanda), Royal Frieslan Campina (Holanda), Dairy Farmers of America (Estados Unidos), Aral Foods (Dinamarca) Saputo (Canadá/ EE UU), Dean Foods (Estados Unidos) y Yill Group (China). Juntas facturaron en 2014, una suma de 141 miles de millones de dólares (Food Manufacture, 2014).

\section{Las organizaciones internacionales de referencia en materia sanitaria y fitosanitaria}

En el proceso de resolución de disputas, asisten a la OMC, con criterios científicos/técnicos, tres instancias que pertenecen al Sistema de las Naciones Unidas. Aunque estas organizaciones desarrollan lineamientos para el comercio internacional, los gobiernos pueden usarlos para su propia legislación interna, de ahí su relevancia.

El Codex Alimentario es una organización internacional creada en 1963 por la FAO y la $\mathrm{OMS}^{6}$ como el mecanismo de referencia sobre inocuidad de alimentos a nivel internacional, que cobró más importancia con el crecimiento del comercio internacional de alimentos, y por su rol en los mecanismos de resolución de controversias relacionadas con la inocuidad de alimentos en la OMC.

Las normas, códigos y estándares establecidas en el Codex son elaboradas por especialistas y expertos en materia de inocuidad de los alimentos. La mayoría de científicos que trabajan en el desarrollo de estas normas son de países industrializados que trabajan en las llamadas "ciencias duras", en áreas muy especializadas. Por lo tanto, examinan los aspectos de inocuidad de los alimentos desde un punto de vista puramente técnico, dejando de lado aspectos sociales y culturales que son importantes en la alimentación.

\begin{tabular}{|l|l|l|}
\hline \multicolumn{2}{|l|}{ CUADRO 1. Comités CODEX. Sus AUSPICIANTES Y sus INTEReses } \\
\hline Comité Codex & País auspiciante & Observaciones \\
\hline Chocolate y cacao & Suiza & $\begin{array}{l}\text { Este país es sede de Nestlé, empresa líder en la } \\
\text { fabricación de chocolate. }\end{array}$ \\
\hline Leche y derivados & Nueva Zelanda & $\begin{array}{l}\text { El 26\% de sus exportaciones provienen del sector } \\
\text { láteo (NZIER, 2010). } \\
\text { Fonterra es la cuarta empresa láctea más grande a } \\
\text { nivel mundial en término de ventas. }\end{array}$ \\
\hline Sanidad de la carne & Nueva Zelanda & $\begin{array}{l}\text { La producción de carne constituye también un rubro } \\
\text { importante en la economía del país. }\end{array}$ \\
\hline Cereales y legumbres & Estados Unidos & $\begin{array}{l}\text { Principal productor y exportador de maíz, soya y trigo. } \\
\text { Las empresas Cargill, ADM y Bunge contralan el } \\
\text { comercio internacional de estas commodities. }\end{array}$ \\
\hline Peces y mariscos & Noruega & $\begin{array}{l}\text { Con un importante sector pesquero. La empresa } \\
\text { noruega Coast Seafood es la primer exportadora de } \\
\text { salmón atlántico a nivel mundial. }\end{array}$ \\
\hline Grasas y aceites & Malasia & Primer productor mundial de aceite de palma. \\
\hline Sopas & Suiza & $\begin{array}{l}\text { La empresa Nestlé es también líder en la elaboración } \\
\text { de sopas. }\end{array}$ \\
\hline $\begin{array}{l}\text { Frutas y } \\
\text { vegetales procesados }\end{array}$ & Estados Unidos & $\begin{array}{l}\text { País que posee una importante industria de } \\
\text { transformación de alimentos. }\end{array}$ \\
\hline
\end{tabular}

Fuente: Codex Alimentario (Lista de Comités Activos). $6 \begin{aligned} & \text { La FAO es la Organización de las Naciones Unidas para la Agricultura y la Alimentación. La OMS es la Organización } \\ & \text { de las Naciones Unidas para la Salud. }\end{aligned}$ 
Las normas son desarrolladas a través de Comités especializados, cada uno de los cuales es auspiciado por un país miembro. En su análisis sobre el Codex Alimentario, Bravo (2002) propone que detrás de los países que auspician un Comité Codex, generalmente hay grupos económicos relacionados con el tema a tratarse, como se muestra en el Cuadro 1, lo que pone en cuestionamiento la "objetividad científica" de las normas desarrolladas.

La Organización Mundial de Sanidad Animal (OIE) es la organización intergubernamental encargada de velar por la sanidad animal a nivel internacional. La OIE desempeña su cometido bajo la autoridad y el control de una Asamblea Mundial de delegados designados por los Gobiernos de los Países Miembros.

En julio de 1998 se elaboró un acuerdo formal de cooperación entre la OIE y la OMC para que participe en los procedimiento de solución de diferencias, que lo hace a través de asesoramientos científico/técnico (Organización Mundial del Comercio, 2007).

En una disputa que se la conoce como "Productos biotecnológicos - Unión Europea", se invitó a la Secretaría de la OIE para que entregue información con respecto al significado de determinados términos y conceptos relacionados con el caso (Organización Mundial del Comercio, 2007)

La OIE está también influenciada fuertemente por el sector privado.

\begin{tabular}{|c|c|c|}
\hline $\begin{array}{l}\text { Gremio privado } \\
\text { internacional }\end{array}$ & Misión & Algunos términos acordados con la $\mathrm{OIE}$ \\
\hline $\begin{array}{l}\text { Federación } \\
\text { Internacional de } \\
\text { Lechería }\end{array}$ & $\begin{array}{l}\text { Es una federación compuesta } \\
\text { por comités nacionales, que } \\
\text { representan de la manera } \\
\text { más amplia posible a las } \\
\text { actividades lecheras de su } \\
\text { país. }\end{array}$ & $\begin{array}{l}\text { Participa en la elaboración y revisión de normas } \\
\text { internacionales de sanidad animal y zoonosis, } \\
\text { necesarias para el comercio internacional de } \\
\text { la leche y los productos lácteos; así como, } \\
\text { en el intercambio de pareceres sobre el } \\
\text { planteamiento por parte de organizaciones } \\
\text { intergubernamentales como la OMS, la FAO y } \\
\text { su organismo subsidiario (Codex Alimentarius) } \\
\text { de las estrategias de vigilancia y control de } \\
\text { enfermedades que pueden tener repercusiones } \\
\text { en el sector lechero y/o en el comercio } \\
\text { internacional. }\end{array}$ \\
\hline $\begin{array}{l}\text { Oficina Permanente } \\
\text { Internacional de la } \\
\text { Carne (OPIC) }\end{array}$ & $\begin{array}{l}\text { Es una organización no } \\
\text { gubernamental que representa } \\
\text { al sector global de la carne y } \\
\text { el ganado como motor vital de } \\
\text { crecimiento para el suministro } \\
\text { sostenible de proteínas } \\
\text { animales seguras, sanas, de } \\
\text { alta calidad y nutritivas, para } \\
\text { garantizar la contribución del } \\
\text { sector como parte esencial de } \\
\text { una dieta sana y sostenible. }\end{array}$ & $\begin{array}{l}\text { Intercambio de pareceres sobre la elaboración y } \\
\text { revisión de normas internacionales en materia } \\
\text { de seguridad sanitaria de los alimentos, sanidad } \\
\text { animal y zoonosis pertinentes para el comercio } \\
\text { internacional de carne y productos cárnicos. } \\
\text { Planteamiento por parte de organizaciones } \\
\text { intergubernamentales como la OMS, la } \\
\text { FAO y su organismo subsidiario (Codex } \\
\text { Alimentarius) de estrategias de vigilancia y } \\
\text { control de enfermedades que pueden incidir en } \\
\text { el sector cárnico y el comercio internacional, en } \\
\text { particular las destinadas a los mataderos. } \\
\text { Sanidad animal y las zoonosis, el bienestar } \\
\text { de los animales y la seguridad sanitaria de los } \\
\text { alimentos. }\end{array}$ \\
\hline
\end{tabular}

\begin{tabular}{|c|c|c|}
\hline $\begin{array}{l}\text { Federación Ecuestre } \\
\text { Internacional }\end{array}$ & $\begin{array}{l}\text { Es la organización que a } \\
\text { nivel internacional gobierna } \\
\text { los deportes ecuestres. Tiene } \\
134 \text { federaciones nacionales } \\
\text { afiliadas y que cuenta con el } \\
\text { apoyo de la empresa privada. }\end{array}$ & $\begin{array}{l}\text { Consulta sobre la elaboración y revisión de } \\
\text { normas zoosanitarias internacionales para el } \\
\text { movimiento internacional de caballos. } \\
\text { Consulta sobre la elaboración y revisión de } \\
\text { reglas, directrices y normas de protección para } \\
\text { el movimiento internacional de caballos. } \\
\text { Intercambio de pareceres sobre } \\
\text { estrategias enfocadas por organizaciones } \\
\text { intergubernamentales como la OMS, la } \\
\text { FAO y la OMC en materia de control de } \\
\text { enfermedades que pueden afectar a caballos de } \\
\text { competición. }\end{array}$ \\
\hline $\begin{array}{l}\text { Comisión } \\
\text { Internacional del } \\
\text { Huevo (IEC) }\end{array}$ & $\begin{array}{l}\text { Representa a la industria del } \\
\text { huevo global }\end{array}$ & $\begin{array}{l}\text { Cooperación para desarrollar y revisar } \\
\text { directrices y normas internacionales sobre la } \\
\text { producción de huevos; el comercio de huevos } \\
\text { y ovoproductos. } \\
\text { Intercambio de opiniones sobre vigilancia } \\
\text { sanitaria en el sector del huevo y el comercio } \\
\text { internacional, a nivel del Códex Alimentario. }\end{array}$ \\
\hline $\begin{array}{l}\text { Consejo } \\
\text { Internacional } \\
\text { Apícola (IPC) }\end{array}$ & $\begin{array}{l}\text { El Consejo Internacional } \\
\text { Avícola (IPC) fue creado para } \\
\text { reunir a líderes de la industria } \\
\text { de aves de corral de todo } \\
\text { el mundo para tratar temas } \\
\text { de comercio, la ciencia, y } \\
\text { mejorar las relaciones entre } \\
\text { las naciones. }\end{array}$ & $\begin{array}{l}\text { Cooperación para desarrollar y revisar } \\
\text { directrices y normas internacionales sobre la } \\
\text { producción avícola. } \\
\text { Intercambio de opiniones sobre vigilancia } \\
\text { sanitaria en el sector avícola a nivel del Códex } \\
\text { Alimentario. }\end{array}$ \\
\hline $\begin{array}{l}\text { Organización } \\
\text { Mundial de Plantas } \\
\text { de Transformación } \\
\text { de Subproductos } \\
\text { Animales WRO }\end{array}$ & $\begin{array}{l}\text { Representa los intereses de la } \\
\text { industria de transformación de } \\
\text { alimentos a nivel mundial. }\end{array}$ & $\begin{array}{l}\text { Desarrollar y revisar directrices y } \\
\text { normas internacionales sobre productos } \\
\text { transformados. } \\
\text { Participación de expertos de cada organización } \\
\text { en las reuniones de las comisiones y grupos de } \\
\text { trabajo de ambas organizaciones. }\end{array}$ \\
\hline $\begin{array}{l}\text { Organización } \\
\text { Mundial de } \\
\text { Agricultores WFO }\end{array}$ & $\begin{array}{l}\text { Organización internacional } \\
\text { de agricultores que } \\
\text { pretende reunir a todas las } \\
\text { organizaciones cooperativas } \\
\text { de productores agrícolas y } \\
\text { nacionales con el objetivo de } \\
\text { desarrollar políticas que les } \\
\text { favorezca. }\end{array}$ & $\begin{array}{l}\text { Intercambio de puntos de vista sobre } \\
\text { la elaboración y la revisión de normas } \\
\text { internacionales, directrices de buenas } \\
\text { prácticas sobre sanidad animal, las zoonosis, } \\
\text { la seguridad sanitaria de alimentos de origen } \\
\text { animal en fase de producción en la medida } \\
\text { en que estos textos repercutan en el comercio } \\
\text { internacional. }\end{array}$ \\
\hline
\end{tabular}

Fuente: Sitios web de la OIE y de las organizaciones citadas.
Consulta sobre la elaboración y revisión de

l movimiento intercion

Intercambio de pareceres sobre

Cooperación para desarrollar y revisa

ovoproductos.

Cooperación para desarrollar y revisar

tercambio de opiniones sobre vigilancia el códex ormas internacionales sobre productos Participación de expertos de cada organización tas reuniones de las comisiones y grupos de Intercambio de puntos de vista sobre ternación y la revisión de nornas prácticas sobre sanidad animal, las zoonosis, a seguridad sanitaria de alimentos de orige a dase de producción en la medida in que estos textos repercutan en el comercio
internacional. movimiento internacional de caballos. 
Se puede ver en este cuadro como los grandes gremios de las distintas ramas de la producción animal participan en la elaboración de normativas que afectan a su sector. Por supuesto, no es 1 sanidad animal ni la inocuidad alimenticia su principal preocupación, sino el de buscar modos para maximizar sus ganancias y fortalecer el comercio internacional de sus productos.

La referencia en materia de sanidad vegetal es la Convención Internacional de Protección Fitosanitaria (CIPF), que elabora guías, disposiciones y da criterios en materia fitosanitaria, especialmente relacionadas con plagas de plantas, que pueden introducirse a través del comercio internacional.

Para la elaboración de estándares en materia fitosanitaria, la CIPF ha establecido acuerdos directos con la industria agroalimentaria, incluyendo entre otros, a la Coalición Internacional del Comercio de Granos y Federación Internacional de Semilla-IPPC (2014a), gremios que tienen una gran capacidad de influencia y cabildeo tanto en la elaboración de normativas nacionales como en el desarrollo de estándares internacionales. La CIPF no incluye en sus procesos de consulta y toma de decisiones a organizaciones campesinas, grupos ambientales o de consumidores; es decir, su opinión no ha sido consultada en el desarrollo de normas que podría afectarles.

El hecho de que las tres instancias consultivas trabajen de manera estrecha con la industria, pone en duda la objetividad de las normas y los estándares desarrollados por ellas, y el mismo objetivo de precautelar la salud humana. Lo dicho puede ilustrarse a través de la siguiente nota de prensa del Consejo Internacional de Avicultura (IPC), donde se muestra la preocupación de este gremio por las medidas que se estaban tomando en relación a la imposición de posibles brotes epidémicas de gripe aviar:

"En un comunicado enviado hoy al Dr. Bernard Vallat, director general de la OIE, el IPC (Consejo Internacional de Avicultura) señala que los casos de influenza generalizadas que afectan a las aves de corral comerciales está causando que muchos paises impongan restricciones comerciales injustificadas, que van en contra de las recomendaciones de la OIE. El IPC sostiene que la manera en que los funcionarios veterinarios en algunos países interpretan las reglas establecidas en el Código Sanitario para los Animales Terrestres "está causando trastornos en el comercio de aves de corral de cría y carne de aves de corral, causando daños potencialmente graves a la producción de aves de corral en su países propios, y poniendo en peligro un elemento esencial de la seguridad alimentaria mundial sostenible" (International Poultry Council, 2015).

Son estos estándares, los que están siendo introducidos en las legislaciones nacionales, no sólo para los productos destinados a la exportación sino también para alimentos que se consumen domésticamente. En todos los casos, se favorece a la producción agroalimentaria a gran escala, a nombre de la sanidad vegetal y animal, la inocuidad alimentaria y la salud humana.

\section{Problemas sanitarios de la actividad agropecuaria gran escala}

Desde el punto de vista sanitario, ninguna gran epidemia ha surgido de estos sistemas campesinos diversificados, en contraste, a los serios problemas sanitarios surgidos de la ganadería industrial, como bien lo señala la organización GRAIN en la siguiente cita:

"En 2009, 200 mil kilos de carne contaminada con una letal bacteria resistente a los antibióticos llegaron hasta los niños de muchas escuelas estadounidenses, antes de que la segunda empacadora más grande del país lograra requisar la carne envenenada. Un año antes, en China, seis bebés murieron y 300 mil más se enfermaron de gravedad con afecciones renales cuando uno de los princion principales productores de lácteos a sabiendas pernitio que se le introdujera un químico industrial a sus reservas de leche. Por todo el mundo, la gente enferma y muere cono nunca antes a causa de la regulaciones, pero pocas de éstas tienen algo que ver con la salud pública" (GRAIN, 2011).
El sistema agroalimentario global es responsable de enfermedades que tuvieron visos de transformarse en epidémicas, como sucedió con la gripe aviar, la gripe porcícola y el mal de la vaca loca. Aunque la gripe aviar se asoció con la cría familiar de pollos en el Sudeste Asiático, y hasta con las aves silvestres migratorias, la enfermedad surgió en la gigante cría industrial de pollos que tiene lugar en esa región del mundo.

La gripe porcicola o conocida también como gripe AH1-N1 fue registrada por primera vez en México, en la comunidad de La Gloria, contigua a Granjas Carroll, subsidiaria de Smithfield Food, donde ya los miembros de esta comunidad -así como de otras contiguas-, habían protestado por las lamentables condiciones de salubridad de estos planteles porcícolas. Al respecto, Ribeiro (2009) señala:

... comunidades enclavadas alrededor de las ciudades de puercos de Granjas Carroll, viven desde hace 14 años con miedo a la contaminación. Respiran día y noche una peste infernal. Las tolvaneras arrastran la fetidez a muchos kilómetros a la redonda. Ellos creen que sus enfermedades respiratorias se deben al trabajo que hace la empresa. Smithfield es la tercera Midland y de Tyson Foods" (Ribeiro, 2009:1)

El mal de las vacas locas o encefalopatías espongiformes transmisibles (EET) se producen por la acción de un prión, que es una proteína alterada que provoca malformaciones en las células del cerebro. Se transmite en seres humanos por el consumo de carne infectada. La enfermedad se desarrolla porque el ganado vacuno es alimentado con restos de otros animales (lo que maximiza las ganancias de la industria cárnica). De esa manera, las vacas pasan de ser rumiantes a carnívoras. Esta práctica empezó luego de la desregularización de la industria de procesamiento de carnes a fines de la década de 1970. Paralelamente surgió un brote de una variedad de la enfermedad humana Creutzfeldt-Jakob, que se la relaciona con el consumo de productos cárnicos contaminados por tejido nervioso central. Esta enfermedad es mortal.

El cambio en la virulencia de Escherichia coli también está relacionada con alteraciones en la dieta del ganado bovino, cuando se pasó de una alimentación basada en pastos a piensos de maíz y soya.

Este conjunto de nuevas enfermedades son el resultado de la cría intensiva de animales que ha incorporado una serie de factores de riesgo con el fin de incrementar la producción. Uno de ellos es el uso de agentes anabólicos. Estos son hormonas (naturales, sintéticas o semi-sintéticos) que influyen en las funciones metabólicas del animal, alterando el balance de nitrógeno en el organismo o que conduce a un incremento de la producción de proteína. Entre las más usadas en la ganadería se incluye a los esteroides y estrógenos que son hormonas gonadales y las que tienen actividad progestacional. La hormona sintética dietilestilbestrol, usada en la ganadería, fue retirada del mercado cuando se encontró que tenía efectos cancerígenos.

El uso de anabólicos es común en Estados Unidos y México; pero se está prohibiendo en la Unión Europea y en todos los países que quieren entrar en el mercado europeo. El uso continuo de carne con residuos de agentes anabólicos puede constituir un problema de salud pública, como muestra un informe de la Dirección de Salud y Protección de los Consumidores de la Comisión Europea, donde se muestra que, al menos seis hormonas de crecimiento usadas en la ganadería, generan diferentes niveles de peligro en la salud humana, siendo al menos una de ellas cancerígena. Estas son hormonas usadas en la producción ganadera en otras partes el mundo (Directorate - General for Health and Consumer, 1999).

Otro de los productos que han sido incorporados en la producción industrial de leche en países como Estados Unidos, es la hormona de crecimiento bovino (rBST), que incrementa la producción de leche. Se trata de un duplicado sintético de la somatotropina bovina, que es un suplemento proteico natural para las vacas. El tratamiento con este producto causa varias 
enfermedades en las vacas, incluyendo la mastitis o infecciones a las ubres, produciendo leche con pus, lo que requiere el uso de grandes cantidades de antibióticos (REDALT, 2005). En varios países la hormona rBST está prohibida, incluyendo Ecuador.

\section{Impactos ambientales de la producción a gran escala}

Según estudios de la FAO (2013), la ganadería (para carne y leche) es una de las principales responsables de la crisis ambiental y climática actual; debido al cambio del uso de la tierra relacionada tanto para crear pastos para el pastoreo, como con las plantaciones industriales de soya, maíz y otros cultivos, para la elaboración de piensos.

Otras fuentes de impactos ambientales son la aplicación de fertilizantes y plaguicidas a los pastos, la producción animal que usa cada vez más combustibles fósiles, especialmente cuando se hace producción estabulada, la descomposición entérica y el procesamiento y transporte internacional. Lo más grave es que se prevé que la producción mundial de carne se duplicará para el 2050 en relación a lo que se producía a inicios del siglo.

En términos de calentamiento global, el ganado vacuno de carne genera cantidades similares de emisiones de gases que producen el efecto invernadero, 2495 millones de toneladas de $\mathrm{CO}_{2}$ equivalentes, lo que significa el $41 \%$ de las emisiones totales del sector ganadero. Les siguen la carne de cerdo con 700 mil toneladas de $\mathrm{CO}_{2}$-equivalentes y la carne de pollo y los huevos con 612 mil toneladas de $\mathrm{CO}_{2}$-equivalentes, cada uno representa entre el $7 \%$ y el $10 \%$ de las emisiones del sector (Gerber et al., 2013).

En un estudio hecho en Nicaragua (Ríos et al., 2012) donde se evaluó la huella hídrica de tres sistemas de producción lechera, se encontró que para producir un litro de leche, se requiere alrededor de 1000 litros de agua, en las lecherías industriales.

\section{Lo que pasa en el Ecuador}

En el Ecuador, la actividad láctea es una de las que genera más valor a nivel de economí familiar y campesina, y ocupa cerca de 620.000 personas entre mano de obra familiar y contratada (Ruiz, 2007).

En los últimos años se ha desarrollado en el Ecuador una gran cantidad de normas sobre medidas sanitarias, inocuidad de los alimentos y buenas prácticas de manufactura, con el fin de cambiar la matriz productiva en el Ecuador?. Estas normas están armonizadas con los estándares emanados por las tres organizaciones internacionales que asesoran a la OMC en materia de sanidad alimentaria, descritas antes. Su aplicación generaría cambios culturales profundos y demandará inversiones prohibitivas para los productores campesinos y artesanales.

Hay varias normas que regulan la producción alimenticia, siendo uno de ellos el registro sanitario, entregado por la ARCSA ${ }^{8}$. Para obtenerlo el productor debe cumplir con una serie de requisitos que son firmados e implementados con un responsable técnico contratado (un ingeniero de alimentos o bioquímico). Esto no sólo que eleva los costos de producción, sino que implica además introducir una serie de cambios en los procesos artesanales, que pueden producir fracturas en las lógicas comunitarias. Estos procedimientos son inspeccionados periódicamente y de manera aleatoria por la agencia de control estatal -ARCSA.

Hay una norma técnica para cada tipo de producto que requiere de registro sanitario. En el siguiente cuadro se resumen algunas normas técnicas específicas para la industria láctea. 7 La propuesta de cambio de matriz productiva para el sector agropecuario, acuícola y pesquero, está compuesto por exportable, diversificación productiva, incrementar la productividad y la calidad, dar valor agregado a la producción, con una produccion intensiva en innovacion, tecnologia y conocimiento y promover la sustentabilidad ambiental.

8 Agencia Nacional de Regulación, Control y Vigilancia Sanitaria.
CUADRO 3. Normas técnicas del INst Ecuatoriano de Normalzación - INEN para Quesos frescos \begin{tabular}{l|l} 
Norma & Número \\
\hline
\end{tabular}

Norma general para leche y productos lácteos

\begin{tabular}{|l|l}
\hline Norma general para leche pasteurizada & NTE INEN 0010:2012 \\
\hline
\end{tabular}

Norma general para quesos frescos no madurados

NTE INEN 1528:2012

Norma general para rotulados de productos alimenticios procesados, envasados y empaquetados

Control microbiológico de los alimentos NTE INEN 022

\begin{tabular}{|l|l} 
Control microbiologico de los alimentos & \\
\hline Detemina de la acidez tituble &
\end{tabular}

Determinación de la acidez titulable cuatoriana NTE INEN 1 529-13

Determinación de grasa láctea a través del índice de Reichert-Meissel

Determinación de calidad métodos de ensayo cualitativos NTE INEN 1500

Normas generales de colores de identificación de tuberías NTE INEN 440

Reglamento de Buenas Prácticas para alimentos

procesados

Alimentos Procesados 27 de mayo 2014

Las mejores prácticas pecuarias de producción de leche

Permiso de funcionamiento de establecimientos sujetos a control sanitario 2014

Reglamento para la cadena de leche y sus derivados. 25 de abril 2013

Reglamento del Sistema de Identificación y Trazabilidad Animal del Ecuador (conocida como areteo)

NTE INEN 0009

Elaboración propia

A través de esta normatividad, se regula con un alto grado de detalle, todos los procedimientos a ser llevados a cabo en la cadena de la lecha y sus derivados, empezando por las unidades productivas, los potreros, el acopio de la leche, el transporte, las instalaciones de transformación de la leche y la comercialización. Muchas de estas normas son imposibles de ser cumplidas. Por ejemplo, en casi todos los casos, se exige el uso de agua potable, tratada, entubada o conectada a la red pública; aun cuando en muchos lugares del país no se cuenta con este servicio. 
Todo este corpus legal ha despertado mucha preocupación, como lo expresa un habitante de la Amazonía: "En Loreto ya nos han dicho que no podremos vender el queso que vendemos a las tiendas después de la fecha que aparece en el registro oficial, ya no puedan aparecer esos productos, nos van a decomisar".

Se ha empezado a aplicar la normatividad sobre medidas sanitarias y buenas prácticas de manera parcial en el Ecuador, y se ha escogido como zona piloto, a la provincia de Loja, particularmente a algunas parroquias queseras de los cantones Loja y Saraguro, donde la mayoría de las familias son productoras artesanales de quesillo.

\section{La situación del pueblo Saraguro}

La economía del pueblo Saraguro se mueve en torno a la elaboración de quesillo y de la agricultura andina. La elaboración del quesillo es una actividad tradicional, que ya está arraigado en las familias y comunidades, pues es tanto parte de la alimentación como fuente de ingresos para cubrir las necesidades básicas de las familias.

Las queserías están casi en su totalidad en manos de pequeños ganaderos, quienes tienen una producción promedio de unos tres quesillos diarios, aunque algunos producen un queso al día. El ordeño se hace manualmente, y cerca del $50 \%$ de la leche es procesada in situ para la elaboración de quesillo que tiene como destino la venta y el autoconsumo. Apenas un 10\% de la producción se vende como leche líquida. Los ganaderos prefieren transformar la leche en quesillo para darle valor agregado, y para evitar su comercialización diaria. Un poblador Saraguro dice:

"La única profesión nuestra es ser ganadero. Hemos vivido así desde niños, cuando había bastantes pastos. Cuando me casé me regalaron unas cabecitas con las que empezamos a trabajar, aunque sea arrendando terrenitos. Asi seguimos trabajando: ordeñando y sacando todas las semanas la cuajada, entregando la cuajada en Loja. Lo único recurso que sacamos de aquí es la cuajada, y as hemos viviendo hasta ahora. Hasta ahora vivo con mi ganadito, ordeñando mis vacas y haciendo cuajada" 10

En el año 2014, se inició una campaña municipal de decomiso de todo quesillo que no tuviera permiso sanitario. De acuerdo al testimonio de pobladores de la zona, en algunas casos hasta se apostaban trabajadores del municipio en la entrada de Loja y en los mercados para exigir el permiso sanitario, y no les dejaban vender ni en el mercado ni en las ferias libres.

exigir el permiso sanitario, y no les dejaban vender ni en el mercado ni en las ferias libres.
Esto desató mucho malestar en la población. Una primera reacción fue la movilización

Esto desató mucho malestar en la población. Una primera reacción fue la movilización
ente al Municipio de Loja por parte de un grupo de productores de quesillo a inicios de julio de 2014, donde manifestaron su preocupación por los graves impactos que esas medidas significaban para ellos. Algunos pequeños productores de queso propusieron que se les declare como "productores artesanales", y que no se les aplicara las mismas normas que a los grandes para su implementación eventualmente tendrán que aplicarlas.

Por eso en agosto de 2015, cuando varios sectores de la sociedad ecuatoriana se movilizaron en torno a una serie de reivindicaciones, las manifestaciones fueron especialmente fuertes en la región de Saraguro y entre otros factores estuvieron motivadas por la preocupación que pes sobre sus habitantes de que el plazo para aplicar las normas sanitarias y de buenas prácticas de manufactura se está acercando, con los impactos económicos que eso significa (Morocho, 2017).

Podría decirse que este es el primer conflicto relacionado con la aplicación de normas sanitarias en el Ecuador.

9 Testimonio recogido el 6 de diciembre 2014, en un taller sobre los impactos de las Buenas Prácticas de Manufactura.

10 Testimonio recabado en enero 2016. San Lucas - Loja, recogida en Bravo (2016).

\section{Conclusiones}

Con las políticas del cambio de matriz productiva en el agro que se quieren introducir en el Ecuador, se está impulsando entre otros aspectos, mejorar la eficiencia, la producción ganadera, homologando la normativa nacional con los estándares establecidos por la OMC y sus organizaciones aliadas. Esto, en un país que es diverso y multicultural.

Aunque estas propuestas se presentan como una forma de elevar los niveles de inocuidad en los alimentos, la calidad de la producción agrícola y ganadera para precautelar la salud de la población, en realidad se trata de aplicar las exigencias que se hacen a la gran industria, con la producción familiar o artesanal a pesar de la gran diferencia en la forma de producir de estos dos sectores.

En la producción agroindustrial los factores de riesgo se multiplican: en la fase agrícola se usan agrotóxicos para el control de pestes y enfermedades, para erradicar malezas y fertilizar el suelo. En los procesos industriales se usa diversos tipos de aditivos sintéticos, para producir productos más atractivos acentuando ciertos sabores y colores; o para alargar su vida en las perchas de los supermercados, etc. Pero es a la producción campesina a la que se le tacha de entregar alimentos que no han pasado las pruebas de inocuidad; o que se elabora sin utilizar las mejores prácticas de manufactura.

Si tales políticas llegaran a tener éxito, significará la sentencia de muerte de la producción campesina tradicional, como ya ha sucedido en otras regiones del orbe, donde la "eficiencia" reemplazó a los pequeños productores de origen campesino.

\section{Bibliografía}

Bravo, Elizabeth. 2016, La regulación empresarial en la producción de alimentos. Impactos en la vida campesina, Universidad Politécnica Salesiana, Abya-Yala. Quito.

2014, "Una visión sobre la bioseguridad en América Latina desde la Ecología Política", en: Toro, C. et al., Ecología Politica de la Bioseguridad en América Latina, Universidad Nacional de Colombia, RALLT, Colección Gerardo Molina, No. 41, Colombia.

Bravo, Ana Lucía. 2002, "Los transgénicos en el Códex Alimentario", en: Bravo, Elizabeth (Ed.), Ayuda alimentaria y organismos transgénicos, RALLT, Quito.

Bryant, Raymond y Bailey, Sinéad. 2005, Third World political ecology. Routledge, Londres

Directorate - General for Health and Consumer. 1999, Growth Hormones in Meat Pose Risk to Consumers Different Levels of Evidence, Boletín de prensa, 17 de mayo de 1999.

Douglas, Mary. 1996, La aceptabilidad del riesgo según las ciencias sociales, Paidós, Barcelona.

Espinosa, A. 2013. Análisis del Acuerdo de Asociación entre Colombia y la Unión Europea: agricultura y medidas sanitarias y fitosanitarias, CEPAL, Serie Estudios y Perspectivas.

FAO. 2013, Mitigación de las emisiones de gases de efecto invernadero en la producción ganadera. Una revisión de las opciones técnicas para la reducción de las emisiones de gases diferentes al $\mathrm{CO}_{2}$. Serie Estudios Producción y Sanidad Animal. 177, Roma.

2002, Sistemas de Calidad e Inocuidad de los Alimentos, Capítulos 2 y 3. Grupo Editorial Dirección de Información de la FAO.

Fernandes Mançano, Bernardo. 2009, "Territorio, Teoría y Política”, en: Flavio, Lozano y Juan Guillermo Ferro (Eds.) Las configuraciones de los territorios rurales en el Siglo XXI-, Editorial Pontificia Universidad Javeriana, Bogotá.

Food Manufacture. 2014, The top 19 dairy companies, en: http://www.foodprocessing.com/top100/top-1002014/, visitado 15 de agosto 2014.

Friedmann, Hanrriet. 2009, Feeding the empire: The pathologies of globalized agriculture. Socialist Register, 41(41), pp. 124-143

Gerber, P.J., et al. 2013. Enfrentando el cambio climático a través de la ganadería - Una evaluación global de las emisiones y oportunidades de mitigación. FAO. Roma. 
GRAIN. 2014, Hambrientos de tierra. Los pueblos indigenas y campesinos alimentan al mundo con menos de un cuarto de la tierra agrícola mundial, documento de análisis.

2001, "La leche en manos de la gente", en: Revista Biodiversidad Sustentos y Culturas, $N^{\circ} 70$, octubre 2011,

Heifer Ecuador. 2014, La agroecología está presente. Mapeo de productores agroecológicos y del estado de

la agroecología en la sierra y costa ecuatoriana, en: http://www.heifer-ecuador.org/wp-content/uploads/ libros/1_La_agroecologia_esta_presente_ES.pdf.

http://www-foodmanufacture.co.uk/World-News/World-s-top-10-dairy-companies

International Poultry Council. 2015, IPC Urges OIE Members to Interpret Rules Properly on AI Trade Bans, en: http://www.internationalpoultrycouncil.org/index.cfm?CFID=4968147\&CFTOKEN=ffbdf22ea9eefc73 F6432F32-A568-A9FB-D45D754D98CF921D, acceso junio 2015.

IPPC. 2014a, List of other organizations with which the IPPC Secretariat cooperates and liaises, disponible en https://www.ippc.int/publications/ippc-secretariats-relationship-other-organizations

2014. Annual Report. 2014. Roma

MAGAP. 2011, Cambio de matriz productiva para el agro, presentación Power Point.

Morocho, Sara. 2017, Sistematización de la aplicación de normas sanitarias en las pequeñas economias indigena de la Parroquia San Lucas - Loja, trabajo de titulación previa la obtención del título de Licenciatura en Gestión para el Desarrollo Local, Universidad Politécnica Salesiana.

NAPPO. 2015, Mensaje del Comité Ejecutivo. Boletín de la NAPPO - mayo de 2015, en: http://www.nappo.org/ es/?sv=\&category=Newsletters\&title=May2015

OMC. s/f, Acuerdo de Medidas Sanitarias y Fitosanitarias, en: https://www.wto.org/spanish/tratop_s/sps_s spsagr_s.htm

2007, Relación con el Codex, la CIPF y la OIE. Comité de Medidas Sanitarias y Fitosanitarias, G/SPS/GEN/775. Pavone, Vicenzo y Flor, Arias. 2012, "Beyond the Geneticization Thesis: The Political Economy of PGD/PGS", en: Spain. Science, Technology and Human Values 37, pp 235-261.

REDALT. 2005, Leche, ingeniería genética y hormona de crecimiento bovino (rbST), en: http://www.ecoportal. net/Eco-Noticias/Leche ingenieria genetica y hormona de crecimiento bovino rbS

Ribeiro, Silvia. 2009, Gripe porcina: la epidemia del lucro, La Jornada, 29 de abril 2009.

Ruiz, P. 2007, "La importancia de la producción de leche en el Ecuador", en: Brassel, F. y F. Hidalgo (Eds.) Libre Comercio y lácteos. Libre comercio y lácteos: la producción de leche en el Ecuador entre el mercado global y la globalización, SIPAE, Ecuador.

Thornsburry Suzanne. 2002, "Sanitary and Phytosanitary Issues: Where does the WTO go from here?", en: Moss, C.B., et. al. (Eds.), Agricultural Globalization Trade and the Environment, Spirnger pp. $269-283$.

\section{Comparación de indicadores ambientales en tres casos de agricultura: tradicional, orgánica y convencional}

Doris Guilcamaigua Paztuñal* Edwin Chancusig Espín**

Resumen

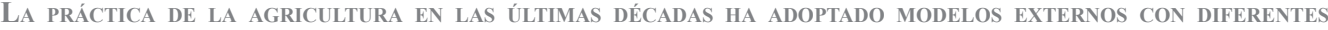

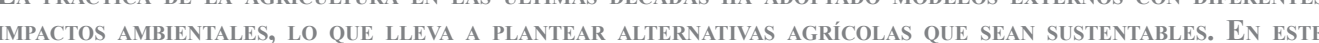
ESTUDIO SE ANALIZA LA SLSTENTABLIDAD DESDE LA PERSPECTIVA AMBIENTAL DE TRES AGROECOSISTEMAS CON DIFERENTES PROPLFSTAS DE PRODLCCIÓY (TRADICIOVAL ORGÁNICO Y CONYFYCLONAL) LBICADOS FY LA PROVINGIA de Chimborazo, Ecuador. La metodología se basa en la Garacterización de sistemas, ge cíchlo de 7

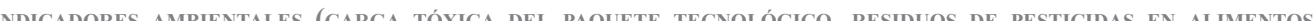
(6) ALORACIÓN DE LA SUSTENTABLIDAD AMBIENTAL.

Palabras claves: agroecosistemas - sustentabilidad - agrobiodiversidad - Chimborazo - indicadores AMBIENTALES - TÉCNICAS ANCESTRALES.

Abstract IMPACTS, WHICH LEAdS TO THE PRoposal OF SUSTAINABLE AGRICULTURAL ALTERNATIVES. THIS STUDY ANALYZES SUSTAINABILITY FROM THE ENVIRONMENTAL PERSPECTIVE OF THREE AGROECOSYSTEMS WITH DIFFERENT PRODUCTION proposals (TRADITIONAL, ORGANIC AND CONVENTIONAL), LOCATED IN THE PROVINCE OF ChIMBORAZO, ECUADOR. THE METHODOLOGY IS BASED ON THE CHARACTERIZATION OF SYSTEMS, THE CALCULATION OF SEVEN ENVIRONMENTAL INDICATORS (TOXIC LOAD OF THE TECHNOLOGICAL PACKAGE, PESTICIDE RESIDUES IN FOOD, PRODUCTIVITY, SOIL QUALITY, WATER QUALITY, AGROBIODIVERSITY, AND ENERGY EFFICIENCY), AND THE ENVIRONMENTAL SUSTAINABILLTY.

KEYWORDS: AGROECOSYSTEMS - SUSTAINABILITY - AGROBIODIVERSITY - CHIMBORAZO - ENVIRONMENTAL INDICATORS - ANCESTRAL TECHNIQUES.

Magister en Proteccion Ambiental. Universidad de las Fuerzas Armadas, Departamento de Energía y Mecánica,

* Docente. Correo electrónico: deguilcamaigua@espe.edu.ec

Universidad Técnica de Cotopaxi, Unidad de Ciencias Agropecuarias y Recursos Naturales. Correo electrónico: edwin.chancusig@utc.edu.ec. 